\title{
Advances in Water Resources
}

\section{Similarity solution of the Boussinesq equation}

\author{
D.A. Lockington $^{\text {a,* }}$, J.-Y. Parlange ${ }^{b}$, M.B. Parlange ${ }^{c}$, J. Selker ${ }^{d}$ \\ ${ }^{a}$ Department of Civil Engineering, University of Queensland, Brisbane 4072, Australia \\ ${ }^{\mathrm{b}}$ Department of Agricultural and Biological Engineering, Cornell University, Ithaca, NY 14853, USA \\ ${ }^{\mathrm{c}}$ Department of Geography and Environmental Engineering, Johns Hopkins University, Baltimore, MD 21218, USA \\ d Department of Bioresource Engineering, Oregon State University, Corvallis, OR 13906, USA \\ Received 31 August 1999; received in revised form 18 December 1999; accepted 19 January 2000
}

\begin{abstract}
Similarity transforms of the Boussinesq equation in a semi-infinite medium are available when the boundary conditions are a power of time. The Boussinesq equation is reduced from a partial differential equation to a boundary-value problem. Chen et al. [Trans Porous Media 1995;18:15-36] use a hodograph method to derive an integral equation formulation of the new differential equation which they solve by numerical iteration. In the present paper, the convergence of their scheme is improved such that numerical iteration can be avoided for all practical purposes. However, a simpler analytical approach is also presented which is based on Shampine's transformation of the boundary value problem to an initial value problem. This analytical approximation is remarkably simple and yet more accurate than the analytical hodograph approximations. (c) 2000 Elsevier Science Ltd. All rights reserved.
\end{abstract}

Keywords: Similarity solution; Boussinesq equation; Aquifer recharge

\section{Introduction}

Groundwater flow in an unconfined aquifer may be approximately modeled by the nonlinear Boussinesq equation, assuming Dupuit's hypothesis of horizontal flow applies [3]. Solutions of the Boussinesq equation are applied in catchment hydrology and baseflow studies (e.g., $[12,14])$ as well as agricultural drainage problems [10] and constructed, subsurface wetlands.

Consider the usual Boussinesq equation for one-dimensional flow in an initially dry, unconfined aquifer:

$\theta_{\mathrm{s}} \frac{\partial h}{\partial t}=K_{\mathrm{s}} \frac{\partial}{\partial x}\left(h \frac{\partial h}{\partial x}\right)$

with

$h(0, t)=h_{0}(t)=\sigma t^{\alpha}$,

$h(x, 0)=0$

and

$h(x \rightarrow \infty, t)=0$,

where $\theta_{\mathrm{s}}$ is the porosity, $K_{\mathrm{s}}$ the saturated hydraulic conductivity, $h$ the height of the water table above a

\footnotetext{
${ }^{*}$ Corresponding author.
}

horizontal impermeable layer and $t$ and $x$ are the time and distance, respectively (see Fig. 1). The parameter $\alpha$ is constant. The objective of this paper is to determine accurate, analytical approximations of Eqs. (1)-(4).

Several approximate analytical solutions already exist for the simple case when $\alpha=0$ such that $h_{0}$ is constant $[4,13]$. Eq. (2) is more general, but more importantly, admits a similarity solution that facilitates accurate solution. Although useful in their own right, such solutions are also valuable in that they provide 'benchmarks' to validate more general numerical solutions. While the physical interpretation of the solutions is straightforward for $\alpha>0$ (boundary water supply head increases with time), the implications of $\alpha<0$ are not so clear. It will be seen later that $\alpha=-1 / 3$ corresponds to the redistribution of a finite quantity of water introduced at $x=0$. For values of $\alpha$ less than this, the solution is nonmonotonic and therefore physically unacceptable in the present situation.

A similarity solution is sought in the form

$h=\sigma t^{\alpha} H(\xi)$,

where

$\xi=x \sqrt{\frac{2 \theta_{\mathrm{s}}(\alpha+1)}{\sigma K_{\mathrm{s}} t^{\alpha+1}}}$

so that Eqs. (1)-(4) become 


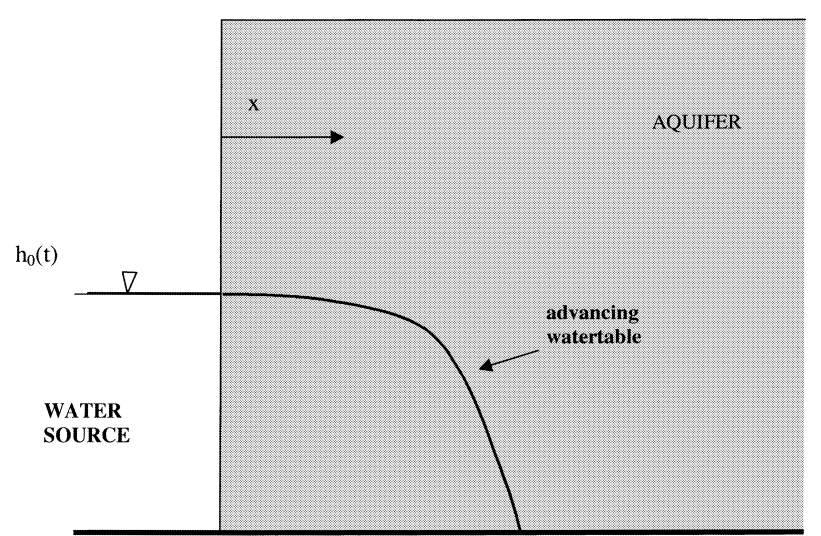

Impermeable horizontal base

Fig. 1. Illustration of physical problem when $h_{0}(t)$ is increasing or constant.

$\frac{\mathrm{d}^{2} H^{2}}{\mathrm{~d} \xi^{2}}+\frac{\xi}{2} \frac{\mathrm{d} H}{\mathrm{~d} \xi}-\lambda H=0$

$H(0)=1$,

$H\left(\xi_{0}\right)=0$.

$\xi_{0}$ is the position of the finite wetting front for an initially dry aquifer and $\lambda=\alpha /(1+\alpha) \in\left(-\frac{1}{2}, 1\right)$.

Chen et al. [2] reformulated (7) as an integral equation using a hodograph transform. Although their equation still requires a numerical solution, it provides an attractive alternative to shooting methods. The hodograph approach of Chen et al. [2] is modified here to obviate the need for numerical iteration for any application of practical interest. A numerical solution to the original equation, which avoids the necessity of 'shooting', can also be obtained by converting the boundary-value problem to a much simpler initial value problem using asymptotic information about the solution at the front, $\xi=\xi_{0}[11]$.

Shampine's method of converting the boundary-value problem, Eqs. (7)-(9), to an initial value problem is used to obtain a simple analytical formula for the watertable elevation in Section 3. This new approximation is compared with the numerical solution of Eqs. (7)-(9) along with the approximations derived from the hodograph formulation.

\section{Analytical hodograph approximation}

Chen et al. [2] introduce the new 'hodograph' variable

$v=-H \frac{\mathrm{d} H}{\mathrm{~d} \xi}$

to transform Eqs. (7) and (8) to
$v(H)=\frac{1}{4}\left[(1+2 \lambda) \int_{0}^{H} \frac{\beta^{2}}{v(\beta)} \mathrm{d} \beta+H \int_{H}^{1} \frac{\beta}{v(\beta)} \mathrm{d} \beta\right]$.

The variable $v$ represents the 'total discharge'. Chen et al. [2] propose a numerical, iterative scheme of (11) with

$v=A H$

and ' $A$ ' constant, as an initial approximation to the solution. This is obtained by considering the asymptotic behaviour of the solution near the front where $H=0$. Chen et al. [2] numerically iterate until a desired convergence is obtained; the convergence rate depends on $\lambda$. However, the leading iteration of their scheme remains analytical and will be further developed here.

Substituting Eq. (12) into the right-hand side of Eq. (11) yields

$v(H) \approx \frac{1}{4}\left(\frac{2 \lambda-1}{2 A} H^{2}+\frac{H}{A}\right)$.

The constant $A$ may be determined by considering Eq. (11) at $H=1$, or

$v(1)=\frac{1+2 \lambda}{4} \int_{0}^{1} \frac{H^{2}}{v(H)} \mathrm{d} H$.

Substituting Eq. (12), we find

$A^{2}=\frac{(2 \lambda-1)^{2}}{16[(2 \lambda-1)-2 \ln ((1+2 \lambda) / 2)]}$.

Now Eq. (13) may be integrated to give the simple analytical approximation

$H(\xi)=\frac{1}{1-2 \lambda}\left[2-(1+2 \lambda) \mathrm{e}^{((1-2 \lambda) / 8 A) \xi}\right]$.

The estimate of the wetting front is then given by

$\xi_{0}=\frac{8 A}{1-2 \lambda} \ln \left(\frac{2}{1+2 \lambda}\right)$.

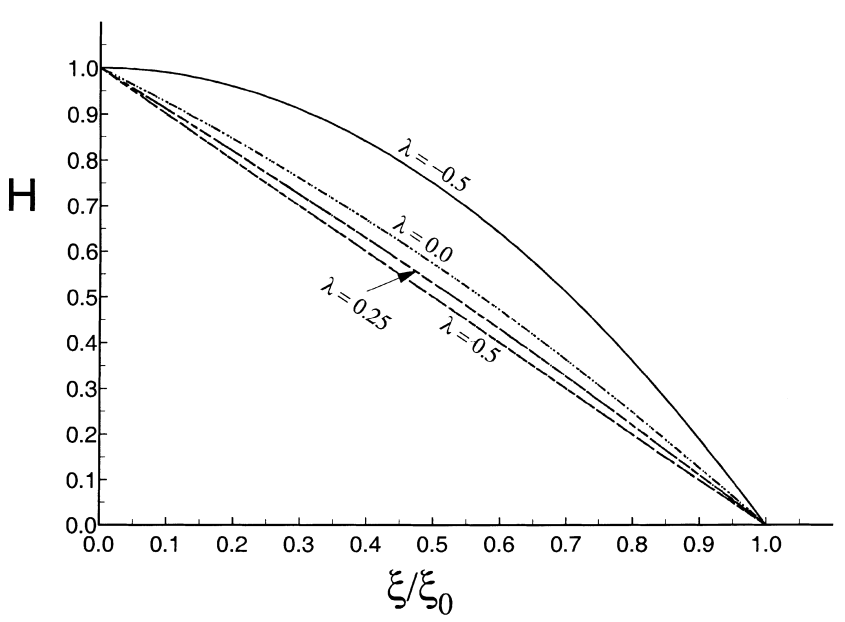

Fig. 2. Numerical solution of Eqs. (7)-(9) for illustrative $\lambda$. 
Convergence of the numerical scheme is slow for $\lambda$ near $-1 / 2$, with the scheme failing for $\lambda=-1 / 2$ (note that Chen et al. [2] avoid the singularity for this limiting value of $\lambda$ by using the corresponding exact solution of Barenblatt et al. [1]). Naturally the analytical approximations (16) and (17) possess this behaviour as well, with an increasing error as $\lambda \rightarrow-1 / 2$ (see Fig. 3).

A variation on the above approach avoids the singularity and results in an improved approximation. With Eq. (10), Eq. (11) can be written as

$v(H)=\frac{1}{4}\left[(1+2 \lambda) \int_{0}^{H} \frac{\beta^{2}}{v(\beta)} \mathrm{d} \beta+H \xi\right]$.

Taking Eq. (12) as the initial approximation to calculate the integral, Eq. (18) yields after integration

$$
\begin{aligned}
H(\xi)= & -\frac{1}{4}\left[\frac{8 A}{1+2 \lambda} \xi-\left(\frac{8 A}{1+2 \lambda}\right)^{2}\right] \\
& +\left[1-\frac{1}{4}\left(\frac{8 A}{1+2 \lambda}\right)^{2}\right] \mathrm{e}^{-((1+2 \lambda) / 8 A) \xi}
\end{aligned}
$$

The coefficient $A$ is found by satisfying Eq. (14), in the form

$v(1)=\frac{1+2 \lambda}{4} \int_{0}^{\xi_{0}} H \mathrm{~d} \xi$,

that is,

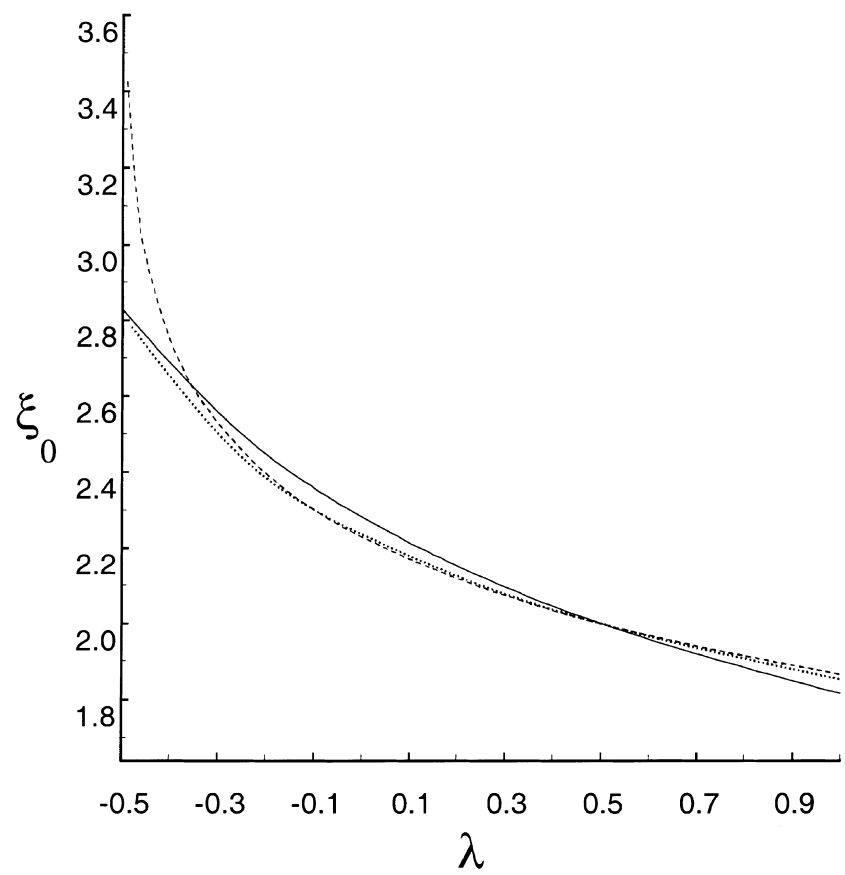

Fig. 3. Estimates of the front position, $\xi_{0}$. Solid line is the numerical solution and the approximation (Eqs. (24) with (26)). The dashed line is Eq. (16) while the dotted line is the corrected hodograph approximation, Eq. (19).
$A=\frac{1}{4} \sqrt{\frac{1+2 \lambda}{1-\xi_{0}^{2} / 8}}$

and $\xi_{0}$ is found implicitly from (19) as

$$
\begin{aligned}
1 & -\frac{\xi_{0}}{2} \sqrt{(1+2 \lambda)\left(1-\xi_{0}^{2} / 8\right)}+\left[(1+2 \lambda)\left(1-\xi_{0}^{2} / 8\right)-1\right] \\
& \times \mathrm{e}^{-\left(\xi_{0} / 2\right) \sqrt{(1+2 \lambda) /\left(1-\xi_{0}^{2} / 8\right)}}=0 .
\end{aligned}
$$

We propose using Eq. (19) without further iteration.

In the following section a different approach enables an accurate numerical solution to be obtained very easily. More significantly it also allows the development of a very simple, yet accurate analytical expansion. This new analytical approximation, together with Eqs. (16) and (19), is then compared with the numerical solution.

\section{Shampine boundary condition: numerical and analytical solutions}

Following [11] we note that at the finite wetting front, $\xi=x i_{0}$, the solution satisfies

$\frac{\mathrm{d} H}{\mathrm{~d} \xi}=-\frac{\xi}{4}$.

It is easy to numerically integrate Eq. (7), satisfying Eqs. (9) and (23), using a fourth-order Runge-Kutta method, for instance, and obtain $H$. Given $\lambda$, the equation is integrated backwards from an arbitrary point $\xi_{0}^{\prime}$ to $H^{\prime}\left(\xi^{\prime}=0\right)$. Scaling invariance of the problem implies that this arbitrary solution curve is related to our desired solution by $H=m H^{\prime}$ and $\xi=m^{1 / 2} \xi^{\prime}$ with $m=1 / H^{\prime}(0)$ (i.e., to satisfy Eq. (8)). Convergence is obtained to at least five significant figures and accuracy confirmed in the case of two exact solutions (see below). The numerical solution is plotted in Fig. 2 for a range of values of $\lambda$.

\subsection{New analytical approximation}

We seek a simple quadratic expansion of the form $H=\alpha\left(\xi_{0}-\xi\right)+\beta\left(\xi_{0}-\xi\right)^{2}$ which satisfies Eq. (8), (9) and (23), or

$H(\xi)=1-\left(2-\frac{\xi_{0}^{2}}{4}\right) \frac{\xi}{\xi_{0}}+\left(1-\frac{\xi_{0}^{2}}{4}\right) \frac{\xi^{2}}{\xi_{0}^{2}}$.

The unknown position of the wetting front $\xi_{0}$ is estimated by forcing Eq. (24) to satisfy the twice-integrated form of Eq. (7)

$\int_{0}^{1} \xi^{2} \mathrm{~d} H=\frac{2}{1+\lambda}$.

This last condition is known to result in accurate approximations for the nonlinear diffusion equation, 
Table 1

Comparison of the analytical approximation, Eqs. (24) and (26) with the Runge-Kutta solution of Eqs. (7)-(9) using Shampine's method

\begin{tabular}{|c|c|c|c|c|c|c|c|c|c|c|}
\hline \multirow[t]{3}{*}{$\xi / \xi_{0}$} & \multicolumn{10}{|l|}{$H$} \\
\hline & \multicolumn{2}{|l|}{$\lambda=-0.5$} & \multicolumn{2}{|l|}{$\lambda=0.0$} & \multicolumn{2}{|l|}{$\lambda=0.25$} & \multicolumn{2}{|l|}{$\lambda=0.5$} & \multicolumn{2}{|l|}{$\lambda=0.9$} \\
\hline & Eq. (24) & Numerical & Eq. (24) & Numerical & Eq. (24) & Numerical & Eq. (24) & Numerical & Eq. (24) & Numerical \\
\hline 1.00 & 0 & 0 & 0 & 0 & 0 & 0 & & 0 & 0 & 0 \\
\hline 0.8 & 0.3600 & 0.3600 & 0.2484 & 0.2483 & 0.2205 & 0.2203 & 0.2 & 0.2 & 0.1764 & 0.1766 \\
\hline 0.6 & 0.6399 & 0.6399 & 0.4727 & 0.4714 & 0.4307 & 0.4300 & 0.4 & 0.4 & 0.3646 & 0.3657 \\
\hline 0.4 & 0.8400 & 0.8400 & 0.6727 & 0.6703 & 0.6307 & 0.6294 & 0.6 & 0.6 & 0.5646 & 0.5665 \\
\hline 0.2 & 0.9600 & 0.9600 & 0.8484 & 0.8461 & 0.8205 & 0.8192 & 0.8 & 0.8 & 0.7764 & 0.7781 \\
\hline 0 & 1 & 1 & 1 & 1 & 1 & 1 & 1 & 1 & 1 & 1 \\
\hline
\end{tabular}

especially if the diffusivity varies rapidly with the dependent variable (e.g., [5-7,9]).

Substitution of (24) yields

$\xi_{0}^{2}=2 \sqrt{1+\frac{12}{1+\lambda}}-2$.

It is interesting to note that Eqs. (24) and (26) give for

$\lambda=\frac{1}{2}, \quad H(\xi)=1-\xi / 2$,

$\lambda=-\frac{1}{2}, \quad H(\xi)=1-\xi^{2} / 8$,

which are in fact the exact solutions of Barenblatt et al. [1] (also see [2,8]). From the calculation of the amount of water entering the aquifer at $x=0$ as $\int_{0}^{\infty} h \mathrm{~d} x$ it is easy to check that $\lambda=1 / 2$ corresponds to a flux at $x=0$ proportional to time; and $\lambda=-1 / 2$ corresponds to the redistribution of a fixed amount of water introduced instantaneously at $x=0$. The analytical approximation, Eqs. (24) with (26), is compared in Table 1 with the numerical solution for several values of $\lambda$. The approximate solution has an error at most on the third significant figure, for $\lambda=0,0.25$, and 0.9 and is indistinguishable from the numerical profiles (Fig. 2). Of course, for $\lambda=0.5$ and -0.5 the analysis being exact provides a check on the accuracy of the numerical results.

Fig. 3 compares the earlier hodograph approximations, Eqs. (16) and (19), with Eq. (24) and the numerical solution for the position of the front $\xi_{0}$ for different $\lambda$. The singularity for Eq. (16) as $\lambda \rightarrow(-1 / 2)$ is clearly seen. The corrected approximation, Eq. (19), does not have the same problem and is reasonably accurate over the range of $\lambda$. Of course, the new analytical result is indistinguishable from the numerical solution, and much simpler than Eq. (19). The approximations are further compared in Table 2 where the full profiles are evaluated for $\lambda=0$. In addition to avoiding the singular behaviour of Eq. (16), Eq. (19) is more accurate. For this value of $\lambda$ the maximum absolute relative error of Eq. (16) is around $6 \%$ while it is around $4 \%$ for Eq. (19). The error in the approximation, Eq. (24), is always less than $1 \%$ with a maximum of around $0.4 \%$.
Table 2

Comparison of analytical approximations, Eqs. (16), (19) and (24), with the exact numerical solution for $\lambda=0$

\begin{tabular}{|c|c|c|c|c|}
\hline \multirow[t]{3}{*}{$\xi / \xi_{0}$} & \multicolumn{4}{|l|}{$H\left(\xi / \xi_{0}\right)$} \\
\hline & \multicolumn{2}{|l|}{ Shampine } & \multicolumn{2}{|c|}{ Hodograph } \\
\hline & Numerical & Eq. (24) & Eq. (16) & Eq. (19) \\
\hline 0.0 & 1.0000 & 1.0000 & 1.0000 & 1.0000 \\
\hline 0.05 & 0.9635 & 0.9644 & 0.9647 & 0.9648 \\
\hline 0.1 & 0.9257 & 0.9273 & 0.9282 & 0.9277 \\
\hline 0.15 & 0.8865 & 0.8886 & 0.8904 & 0.8888 \\
\hline 0.2 & 0.8461 & 0.8484 & 0.8513 & 0.8482 \\
\hline 0.25 & 0.8042 & 0.8068 & 0.8108 & 0.8058 \\
\hline 0.3 & 0.7610 & 0.7636 & 0.7689 & 0.7617 \\
\hline 0.35 & 0.7163 & 0.7189 & 0.7254 & 0.7161 \\
\hline 0.4 & 0.6702 & 0.6727 & 0.6805 & 0.6689 \\
\hline 0.45 & 0.6227 & 0.6249 & 0.6340 & 0.6203 \\
\hline 0.5 & 0.5738 & 0.5757 & 0.5858 & 0.5702 \\
\hline 0.55 & 0.5233 & 0.5249 & 0.5359 & 0.5187 \\
\hline 0.6 & 0.4713 & 0.4727 & 0.4843 & 0.4659 \\
\hline 0.65 & 0.4179 & 0.4189 & 0.4308 & 0.4118 \\
\hline 0.7 & 0.3629 & 0.3636 & 0.3755 & 0.3564 \\
\hline 0.75 & 0.3064 & 0.3068 & 0.3182 & 0.2998 \\
\hline 0.8 & 0.2483 & 0.2484 & 0.2589 & 0.2420 \\
\hline 0.85 & 0.1886 & 0.1886 & 0.1975 & 0.1831 \\
\hline 0.9 & 0.1273 & 0.1272 & 0.1339 & 0.1231 \\
\hline 0.95 & 0.0645 & 0.0644 & 0.0681 & 0.0621 \\
\hline 1.0 & 0.0000 & 0.0000 & 0.0000 & 0.0000 \\
\hline
\end{tabular}

\section{Conclusion}

Approximate analytical solutions of the self-similar form of the Boussinesq equation are sought when the boundary head varies with time. The numerical hodograph method of Chen et al. [2] is modified to produce an analytical approximation. The result is sufficiently accurate for practical purposes, but requires the solution of a nonlinear, algebraic equation.

A simpler and more accurate analytical approximation is obtained by forcing a simple quadratic profile to satisfy Shampine's condition at the front of the advancing watertable and an integral condition derived by twice integrating the governing equation. This new approximation is extremely accurate (yet completely tractable), even yielding known exact results for partic- 
ular values of $\lambda$, a parameter related to the time-variation of the boundary head.

\section{References}

[1] Barenblatt GI, Entov VM, Ryzhik VM. Theory of fluid flows through natural rocks. Kluwer Academic Publishers: Dordrecht, 1990.

[2] Chen Z-H, Bodvarssan GS, Witherspoon PA, Yortsos YC. An integral equation formulation for the unconfined flow of groundwater with variable inlet conditions. Trans Porous Media 1995; 18:15-36.

[3] de Marsily G. Quantitative hydrogeology. Academic Press: London, 1986.

[4] Lockington DA. Response of unconfined aquifer to sudden change in boundary head. J Irrig Drainage Engng 1997;123:24-7.

[5] Parlange J-Y. On solving the flow equation in unsaturated soils by optimization: Horizontal infiltration. Soil Sci Soc Amer Proc 1975;39:415-8.

[6] Parlange MB, Prasad SN, Parlange J-Y, Römkens MJM. Extension of the Heaslet-Alksne technique to arbitrary soil water diffusivities. Water Resources Res 1992;28:2793-7.
[7] Parlange J-Y, Barry DA, Parlange MB, Hogarth WL, Haverkamp R, Ross PJ, Ling L, Steenhuis TS. New approximate analytical technique to solve Richards equation for arbitrary surface boundary conditions. Water Resources Res 1997;33: 903-6.

[8] Parlange J-Y, Hogarth WL, Govindaraju RS, Parlange MB, Lockington DA. On an exact analytical solution of the Boussinesq equation. Trans Porous Media 2000, in press.

[9] Parslow J, Lockington D, Parlange J-Y. A new perturbation expansion for horizontal infiltration and sorptivity estimates. Trans Porous Media 1988;3:133-44.

[10] Perrochet P, Musy A. A simple formula to calculate the width of hydrological buffer zones between agricultural plots and nature reserve areas. Irrig Drainage Syst 1992;6:69-81.

[11] Shampine LF. Some singular concentration dependent diffusion problems. ZAMM 1973;53:421-2.

[12] Szilagyi J, Parlange MB. Baseflow separation based on analytical solutions of the Boussinesq equation. J Hydrol 1998;204:251-4.

[13] Tolikas PK, Sidiropoulos EG, Tzimopolous CD. A simple analytical solution for the Boussinesq one-dimensional groundwater flow equation. Water Resources Res 1984;20:24-8.

[14] Troch PA, De Troch FP, Brutsaert W. Effective water table depth to describe initial conditions prior to storm rainfall in humid regions. Water Resources Res 1993;29:427-34. 